\title{
The many facets of stress urinary incontinence
}

\author{
Lesley Carr, MD, FRCSC; ${ }^{*}$ Jacques Corcos, MD, FRCSC ${ }^{\dagger}$
}

*Associate Professor, University of Toronto, Department of Surgery, Division of Urology, Sunnybrook Health Sciences Centre, Toronto, ON; 'Professor of Surgery (Urology), McGill University, Montreal, QC

Cite as: Can Urol Assoc J 2012;6(5):S113. http://dx.doi.org/10.5489/cuaj.12233

$\mathrm{T}$ he $2^{\text {nd }}$ Annual Canadian Urology Forum (2012) focused on decisions and controversies in the management of two distinct clinical entities: stress urinary incontinence (SUI) in women; and lower urinary tract symptoms (LUTS) in men.

Both sessions involved a distinguished panel of Canadian and international experts and combined the best evidence-based medicine with the considerable clinical experience of the faculty. The overall learning objectives of this session were to:

- Describe the pathophysiology of SUI in female patients;

- Determine appropriate approaches to treatment-related complications or failure in female patients who are experiencing SUI; and

- Measure patient outcomes and define successful treatment of SUI.

To that end, faculty members were asked to cover six individual topics in SUI. Dr. John DeLancey, Norman F. Miller Professor of Gynecology and Professor of Urology at the University of Michigan Medical School, discussed the causes of SUI. Dr. Philippe Zimmern, Professor of Urology at the University of Texas Southwestern Medical Center at Dallas, provided an overview of what constitutes an appropriate workup for a woman with SUI. Dr. J. Christian Winters, Professor of Urology and Gynecology at the Louisiana State University Health Sciences Centre, reviewed the relevant evidence in presenting a rational approach to selecting a surgical intervention for women with SUI. Dr. Victor Nitti, Professor of Urology and Obstetrics and Gynecology and the Vice Chairman of the Department of Urology at New York University (NYU) Langone Medical Center, was tasked with reviewing the potential complications of midurethral slings and presenting options for managing these complications. Dr. E. Ann Gormley, Professor of Surgery (Urology) and the Program Director of the Urology Residency at Dartmouth-Hitchcock Medical Center in Lebanon, New Hampshire, discussed the appropriate management of women with a failed midurethral synthetic sling. Finally, Dr. Zimmern led a thought-provoking discussion of what constitutes treatment success for patients with SUI. The following pages feature brief summaries of each of the above topics.

At the conclusion of these presentations, the faculty and participants interacted to discuss a case study detailing the appropriate management of a woman with complicated SUI. The highlights of this discussion are also captured in the following pages.

The presentations and discussions on SUI at the 2012 Canadian Urology Forum were highly informative and clinically valuable to those in attendance. We hope that by providing the following summaries in this supplement format, these sessions can also provide valuable information to other health-care professionals in Canada and around the world.

Competing interests: Dr. Corcos is an ongoing paid consultant with Astellas Canada. Dr. Corcos and Dr. Carr have received speaker fees, educational grants and/or travel assistance from Astellas Canada within the last two years.

Correspondence: Dr. Lesley K. Carr, Division of Urology, Room MG501, Sunnybrook Health Sciences Centre, 2075 Bayview Ave, Toronto, ON M4N 3M5; fax: 416-480-5116; lesley.car@@sunnybrook. ca; Dr. Jacques Corcos, Hôpital Général Juif - Sir Mortimer B. Davis Urologie E-944, 3755 Ch De La Côte-Sainte-Catherine, Montreal, QC H3T 1E2; icorcos@uro.jgh.mcgill.ca 